\title{
LA CONSTRUCCIÓN MEDIÁTICA DEL ESTIGMA DE PROSTITUTA EN ESPAÑA
}

\section{Resumen}

Los medios construyen un discurso que simplifica la realidad de la prostitución y estigmatiza a quien la ejerce. Este artículo parte de un análisis del discurso de la representación de la prostitución en los periódicos El País y ABC, desde la Transición española al 2012. A pesar de ser ideológicamente opuestos (conservador y católico el ABC, e identificado con la ideología de centroizquierda próxima al PSOE El País), en ambos está presente el estigma. Es un estigma construido de forma diferente, ya que la imagen de la prostituta está articulada en torno a la moral en $\mathrm{ABC}$ y alrededor del ideario progresista en El País, pero que cuenta con un mismo punto de partida y una confluencia final. En ambos, la mujer que se prostituye es prostituta antes que mujer y persona y ambos acabarán coincidiendo en reducir la representación de la prostituta a la «hipervíctima» desde una perspectiva abolicionista.

Palabras clave: Prostitución, estigma, estudios feministas, Comunicación y Género

\section{Resumo}

\section{A construção mediática do estigma da prostituta em Espanha}

Os media constroem um discurso que simplifica a realidade da prostituição e estigmatiza quem a exerce. Este artigo parte de uma análise do discurso da representação da prostituição nos jornais El País e $A B C$, desde a transição espanhola até 2012. Apesar de serem ideologicamente opostos (conservador e católico o $A B C$ e identificado com a ideologia de esquerda do PSOE, o El País), em ambos está presente o estigma. É um estigma construído de forma diferente, já que a imagem da prostituta é articulada em torno da moral no $A B C$ e em torno da ideologia progressista no El País, mas que tem o mesmo ponto de partida e uma confluência final. Em ambos, a mulher que se prostitui é prostituta antes de ser mulher e pessoa e ambos acabarão por coincidir na redução da representação da prostituta à de «hipervítima» numa perspetiva abolicionista.

Palavras-chave: Prostituição, estigma, estudos feministas, Comunicação e Género

\footnotetext{
* Universidad Estatal de Milagro, Ecuador/Universidad de Santiago de Compostela, España.

Dirección postal: Universidad Estatal de Milagro. Cdla. Universitaria Km. 1.5 vía Milagro Km. 26. Edificio de Postgrado. Milagro. Ecuador.

Correo electrónico: belenpunhal@gmail.com

** Universidad Estatal de Milagro, Ecuador

Dirección postal: Universidad Estatal de Milagro. Cdla. Universitaria Km. 1.5 vía Milagro Km. 26. Edificio de Postgrado. Milagro. Ecuador.

Correo electrónico: tamarit03@gmail.com
} 


\begin{abstract}
The Mediatized Construction of the Stigma against the Prostitute in Spain

The media construct a speech that simplifies the reality of prostitution and stigmatizes those who exercise it. This article is based on an analysis of the discourse of the representation of prostitution in the newspapers El País and ABC, from the Spanish transition to 2012. Despite being ideologically opposed (conservative and Catholic $A B C$, and identified with the progressive ideology next to PSOE El País), in both the stigma is present. It is a stigma constructed differently, since the image of the prostitute is articulated around the morality in $\mathrm{ABC}$ and around the progressive ideas in El País, but with the same starting point and a final confluence. In both, the woman who prostitutes herself is a prostitute before being a a woman and a person and both will coincide in reducing the representation of the prostitute to the «hypervictim» from an abolitionist perspective.
\end{abstract}

Keywords: Prostitution, stigma, feminist studies, Communication and Gender

\title{
Introducción. Las prostitutas como las Otras
}

El estigma es la marca con la que identificamos y excluimos al Otro, a quien es o está en el mundo de forma diferente a Nosotros/as, a quien no forma parte del colectivo en el que nos integramos. Las mujeres han sido y son marginadas en una sociedad marcada por el patriarcado y el sexismo. Esta discriminación se incrementa en el caso de las mujeres prostitutas que, en las últimas décadas, han sido mayoritariamente inmigrantes en España. Sufren la interseccionalidad de discriminaciones marcadas no sólo por el género, sino también por su clase social, su condición de inmigrante, su origen étnico o nacional y el estigma que, históricamente, ha marcado a las mujeres que trabajan en la prostitución. Los medios de comunicación, como veremos en este artículo, han jugado un papel clave en la construcción de este estigma. De ahí que, en la investigación que ha servido de base para el mismo, se haya decidido analizar la representación de las mujeres en dos de los principales periódicos españoles.

El de las mujeres que ejercen la prostitución es uno de los colectivos sobre los que recae mayor censura moral (Oselin 2009), una censura que sirve como grillete para el ejercicio del control de la sexualidad femenina. El estigma de "puta», de hecho, se aplica no sólo a las mujeres que trabajan en la prostitución sino a todas aquellas que no se ciñen a los patrones convencionales patriarcales en las relaciones sexuales y de pareja. En las sociedades patriarcales, una "puta» representa no sólo a la mujer que vende sus servicios sexuales, sino también a todas aquellas que evidencian su deseo erótico y ejercen activamente su sexualidad, a todas las que incumplen el contrato sexual previo al contrato social del que nos hablan los pensadores ilustrados. Tal y como explica Caruncho, siguiendo a Pateman, «el contrato sexual, el que firman los hombres entre sí para excluir a las mujeres, es previo al contrato social y garantiza la exclusión de las mujeres de la convención 
de lo social [...], de la esfera de lo público» (Caruncho 2010, 21). ${ }^{1}$ Los hombres no hubiesen podido dominar y dedicarse en exclusiva al espacio público (contrato social) si no hubiese quien cumpliese con las funciones de sustento y cuidado en el hogar (contrato sexual).

El estigma es estratégico para la construcción identitaria del «Nosotros», en oposición al «Otro». Las mujeres, tal y como señalan autoras como Juana Gallego (2010), son representadas en los medios de comunicación como «las Otras» frente al «Nosotros», como protagonista mayoritario masculino en los medios. Si las mujeres son las Otras, las prostitutas son las «Otras de las Otras». En el imaginario social y mediático sobre la prostitución, la construcción de la prostituta como la Otra no sólo se basa en la oposición entre la mujer que sigue el ideario patriarcal en el comportamiento sexual y la que no, sino también en oposiciones de clase social (Rosembergv y Andrade 1999; Andrade 2004), ${ }^{2}$ étnicas o de procedencia nacional (Pitman 2002; Janzen et al. 2013; Fong, Holroyd y Wong 2013; Stenvoll 2002; van San y Bovenkerk 2013; Ribeiro et al. 2007). ${ }^{3}$

Esto nos lleva a una reflexión sobre el propio concepto de estigma y su naturaleza interseccional. El estigma es, para Goffman (2006), un atributo profundamente desacreditado que relega a la persona al ostracismo social. Esta concepción, que se nutre del interaccionismo simbólico, se basa en la interacción entre los individuos y la interpretación de estos procesos de comunicación y ha sido criticada por no tener en cuenta las estructuras sociales que son causa de que las interacciones se desenvuelvan de determinado modo (Rizo 2011). Scambler (2007), en con-

Carole Pateman se incluye ideológicamente en el feminismo radical, que considera la prostitución como una manifestación de la violencia de género. Frente a esta concepción, surgen desde el feminismo posiciones contrarias al ideario abolicionista y que defienden la legalización de la prostitución (Ribeiro et al. 2007).

2 De su análisis de la cobertura mediática realizada por la Folha de São Paulo sobre la prostitución infanto-juvenil en la década de los 1990, Andrade (2004) y Rosemberg y Andrade (1999) concluyen que se esconde un discurso estigmatizador que marca a las niñas y adolescentes de la calle con la prostitución, al igual que graba a los niños y adolescentes de la calle con la criminalización.

Pitman (2002) y Janzen et al. (2013) hacen referencia a la estigmatización de la prostituta en la prensa canadiense, no sólo por el ejercicio del trabajo sexual sino por su origen étnico, en el caso de las mujeres indígenas que ejercen la prostitución. Fong, Holroyd y Wong (2013) detectan también estigmatización en Hong Kong con las mujeres que se prostituyen procedentes de la China continental. Stenvoll (2002) observa el refuerzo de la estigmatización, en función del origen étnico, en la cobertura mediática de la prostitución de mujeres rusas en el norte de Noruega desde 1990 a 2001. Por su parte, van San y Bovenkerk (2013) aluden a la percepción del proxeneta hijo de familias migradas como un peligro para la sociedad holandesa. Sacramento y Ribeiro (2014), en referencia a la investigación en la que participan sobre la estigmatización de las mujeres que se prostituyen en las regiones transfronterizas entre Portugal y España, mayoritariamente de América del Sur y más en concreto de Brasil (Ribeiro et al. 2007), señalan que en la construcción de atributos estigmatizantes es evidente la interseccionalidad de las categorías de género, «raza» y nacionalidad. 
secuencia, insiste en las relaciones de clase, estatus, orden, género y étnicas tras la configuración del estigma. En esta interseccionalidad de las estructuras presentes tras el estigma pone el foco Juliano (2005), quien señala que en la gran estigmatización que padecen las trabajadoras sexuales confluyen prejuicios de base religiosa, étnicos y condicionamientos de clase.

El estigma va, así mismo, de la mano del concepto de «pánico moral», entendido este como el miedo a una persona o grupo considerado como amenaza (Treviño 2009). Este miedo, como apunta Treviño (2009), tiene un carácter irracional, es decir, responde a ansiedades generalizadas que son desplazadas a un grupo social determinado, dejando así a un lado sus causas reales (Soderlund 2002). En el caso de la prostitución, este pánico nace del sentimiento de amenaza al «Nosotros/as», tanto a los preceptos morales que fundamentan ese «Nosotros/as» como a la construcción subjectiva de nuestro «yo» en oposición a lo que se considera abyecto. Janzen y compañeras (2013) indican, al respecto, que el rechazo al Otro nace, en realidad, del desprecio a lo que consideramos abyecto en nosotros/as mismos, aquello que, desde pequeños/as, nos enseñan a rechazar de nosotros/as, para construir nuestra identidad normalizada.

El pánico moral es, por lo tanto, construido y se alimenta de la gran distancia entre la realidad y las exageraciones mediáticas que se produce en la representación de la prostitución. ${ }^{4}$ De este alejamiento entre la realidad y la construcción mediática surge la aplicación, a este contexto, del concepto de mito, que entendemos aquí según lo comprende Barthes (1957), como un habla, un sistema de comunicación y un mensaje despolitizado, que no surge de la naturaleza de las cosas sino de su reconstrucción histórica. O`Neill et al. (2008) indican la existencia de tres mitos dominantes en los que se basan las respuestas jurídicas contemporáneas a la prostitución. Mitos que están estrechamente relacionados con la estigmatización de la prostitución como desviación social (Benoit y Hallgrinsdottir 2011) surgida del choque entre actores inscritos en distintas perspectivas morales (Sacramento y Ribeiro 2014).

1) La expansión de enfermedades de transmisión sexual (ETS). Que las personas que ejercen la prostitución estén bien informadas sobre las ETS no disipa ese temor. Es más, el mito construye a la prostituta como amenaza para la salud, pero no al cliente, que es quien demanda dichas prácticas sexuales de riesgo. ${ }^{5}$

De la ruptura de la distancia con la que se mira surge un importante instrumento para la quiebra de los estereotipos. Alexandra Oliveira (2011) intenta romper con los estereotipos que minan la prostitución de calle mediante una investigación en la que ella misma convivirá, desde octubre de 2002 hasta noviembre de 2007, con las personas que ejercen la prostitución en este espacio. Y de la investigación extraerá conclusiones que se alejan de algunos de los mitos mediáticos con los que se estigmatiza la prostitución, como la victimización o el presentarlas en exclusiva como personas sin capacidad de agencia, de actuación.

5 Se indica en el artículo de $\mathrm{O}^{\prime}$ Neill et al. (2008), contextualizado en el ejercicio de la prostitución 
2) La identificación del trabajo sexual como un comportamiento antisocial que rompe con las normas más básicas del civismo en el espacio público (ruidos, basura, relaciones sexuales en público, profilácticos en la calle...). En este caso, la sanción social recae de nuevo en la prostituta pero no en quien compra sus servicios y alimenta el comercio sexual.

3) La prostitución como violencia de género, en el que intervienen agentes (proxenetas, traficantes, compradores de sexo...) que infringen a las prostitutas daños físicos y psicológicos. Sin embargo, este discurso se contradice con la existencia de otras prácticas de control que las criminalizan (en algunos municipios españoles, multas que castigan el ejercicio de la prostitución en la calle).

Los medios de comunicación son, pues, poderosos agentes en la construcción de los mitos que refuerzan algunos de los principales estigmas que pesan sobre quien ejerce la prostitución. La investigación de la que nace este artículo se elabora desde el ámbito disciplinario de las Ciencias de la Comunicación y surge precisamente de la consideración de los medios como importantes agentes de socialización y de la confluencia de las teorías feministas y de género y las teorías de la comunicación. Más en concreto, de aquellas teorías de la comunicación que nacen de la consideración de los medios como activos constructores de la realidad social y sustento de las relaciones de poder en las que se basa dicha construcción, además de transmisores de ideología, también de ideología sexista.

Los medios, siguiendo la metáfora con la que Gaye Tuchman $(1983)^{6}$ introduce en las Ciencias de la Comunicación las teorías del framing surgidas del interaccionismo simbólico de Goffman, son hoy en día las ventanas a través de las cuales construimos nuestra visión del mundo. Una construcción que no es ajena al poder en una sociedad en la que, según Teun A. van Dijk (2009), éste es fundamentalmente persuasivo y está en buena parte basado en el acceso preferencial al control del discurso público por parte de las élites ${ }^{7}$. Élites representadas por lo que Amparo Moreno denomina como «el arquetipo viril masculino», «varones adultos de los grupos dominantes que representan papeles sociales vinculados al ejercicio

de calle en las ciudades británicas, que el hecho de que las trabajadoras sexuales acostumbren a estar bien informadas sobre su salud, incluso con tasas relativamente bajas de ETS, no amortigua las representaciones de los medios que las relacionan a ellas, y no a los clientes, con la realización de prácticas sexuales de riesgo

6 Gaye Tuchman (1983) compara los contenidos que los medios elaboran con aquella imagen que vislumbramos a través de una ventana. Lo que vemos por la ventana estará determinado por el cristal, el propio marco que limita y delimita lo que conseguimos observar del exterior, e incluso la posición de quien esté mirando.

Teun A. van Dijk (1990), no obstante, se aleja de una concepción omnímoda del poder y de la ideología. Considera que su efecto en las personas, el efecto, por ejemplo, del discurso transmitido por los grandes medios de comunicación, se ve matizado por los esquemas cognitivos previos presentes en el receptor o receptora. 
del poder» (Moreno 1998, 32). Las prostitutas quedan, en los medios, relegadas a los márgenes despoderados, márgenes en los que se encasilla en general a las mujeres como las Otras, y de forma especialmente incisiva, a las prostitutas como «las Otras de las Otras».

\section{El contexto español}

En las últimas cuatro décadas, la prostitución ha cambiado varias veces de cara en España. De ser ejercida por mujeres mayoritariamente autóctonas, en la década de los 1970, se asienta en la década de los 1980 el perfil de la mujer drogodependiente y, ya en la de los 1990, se introducen de modo mayoritario las mujeres inmigrantes. En los últimos años, sin embargo, a raíz de la crisis económica, se ha incrementado el número de mujeres españolas que buscan una salida en la prostitución (Puñal 2015).

La prostitución, por lo tanto, aparece siempre cruzada en España por la interseccionalidad de márgenes varios como el género, la pobreza, las drogodependencias, la orientación sexual o la inmigración. Encontrar datos fiables es complejo, porque el ejercicio de la prostitución no está legalizado como trabajo y, además, la condición de inmigrantes en situación irregular ha hecho que una parte importante de las mujeres que trabajan en el sector lo hagan en la clandestinidad y huyendo de los controles policiales.

En el II Plan Integral de Lucha contra la Trata de Mujeres y Niñas con Fines de Explotación Sexual (2015-2018) se recogen datos aproximativos procedentes de varias fuentes (Ministerio de Sanidad, Servicios Sociales e Igualdad 2015). La prostitución supone en España el 0,35\% del PIB, lo que equivale a unos 3700 millones de euros, según cifras publicadas por la prensa española (El País 2014), apoyándose en datos del Instituto Nacional de Estadística. El número de mujeres que trabajan en la prostitución varía, así mismo, de modo notable según la fuente: desde las 45000 que estima el Centro de Inteligencia contra el Crimen Organizado hasta las 400000 que se calculan en el Informe sobre la situación de la prostitución en España, del 2007, o las 600000 de las que ha informado, en sus emisiones radiofónicas, la cadena SER (Brufao 2011). De prostitución masculina y prostitución transgénero, se sabe aún menos (Puñal 2015).

Dispares, hasta niveles extremos, son también los datos sobre el porcentaje de mujeres víctimas de trata. Para la exdirectora del Instituto de la Mujer de España, Laura Seara, lo son el $90 \%$ de las mujeres que se prostituyen (Puñal 2010). Estudios realizados desde posturas pro legalización reducen notablemente este porcentaje. En una investigación elaborada por la socióloga Laura Oso (2004), de las 45 mujeres que se habían entrevistado, sólo dos habían llegado a España engañadas.

Los cambios en el rostro de la prostitución española están estrechamente unidos a los flujos económicos y poblacionales neoliberales. La prostitución se convierte en el destino de una parte de las mujeres que emigran a España huyendo de 
la situación de empobrecimiento de sus países de origen. El Informe de la Ponencia sobre prostitución en España expone que «la oferta es de mujeres extranjeras muchas de ellas en situación de inmigración ilegal» (Cortes Generales de Estado 2007, 41). En el II Plan Integral de Lucha contra la Trata de Mujeres y Niñas con fines de Explotación Sexual (2015-2018), se reconoce que la feminización de la pobreza y la explotación del deseo de migrar convierten a las mujeres en víctimas más vulnerables (Ministerio de Sanidad, Servicios Sociales e Igualdad 2015). Clara Pérez (2003) habla, así mismo, de la feminización de los flujos migratorios y contrasta la invisibilización de las mujeres inmigrantes en España, a la que vincula fundamentalmente al sector de los servicios domésticos y del cuidado, con la hipervisibilización mediática de aquellas inmigrantes que se dedican al trabajo sexual. Y es que, en este flujo norte-sur, a las mujeres se les han adjudicado, en el territorio español, los trabajos más precarios (como el servicio doméstico o la agricultura) y más estigmatizados, como es el caso de la prostitución. ${ }^{8}$

\section{Estado de la cuestión}

Los estudios sobre Comunicación y Género han experimentado un importante auge en España desde el inicio del nuevo milenio. Sin embargo, un análisis del estado de la cuestión de lo realizado en este campo muestra que falta ahondar en la interseccionalidad de la discriminación de género con otros rostros de la discriminación. De ese vacío, dada la escasez de estudios en esta línea, nace la investigación de la que parte este artículo (Puñal 2015).

Las reflexiones sobre el tratamiento mediático de la prostitución que localizamos en la fase exploratoria de esta investigación se caracterizaban por ser secundarias en estudios con otros objetivos prioritarios de análisis, como por ejemplo, la inmigración.

Así mismo, la reflexión sobre los medios apareció de manera tangencial en trabajos de carácter abolicionista y pro-legalización. Desde una perspectiva abolicionista, los medios de comunicación son criticados por transmitir la cultura de la prostitución como realidad inevitable por hacer uso de conceptos como autodeterminación y libertad sexual y promocionar a través de ellos la industria del sexo (Alba 2006) y por actitudes como la publicación, en la prensa española, de anuncios de sexo de pago, mientras en sus contenidos informativos se critica la

En el Estudio sobre la situación laboral de la mujer inmigrante en España (Iglesias et al. 2015), se afirma la existencia de un amplio bloque social de precariedad, formado por el $88 \%$ de la población femenina inmigrante, y caracterizado por el ejercicio de trabajos de cuidados y de ocupaciones manuales de cualificación básica, con salarios inferiores a los mil euros mensuales. No se hace referencia, en este estudio, a las mujeres que se dedican a la prostitución, pues ésta no está reconocida como trabajo en España. 
explotación sexual y la trata y el tráfico de mujeres. ${ }^{9}$ Desde las posiciones a favor de la legalización, se considera que los medios presentan a la prostitución como mayoritariamente forzada y, desde preceptos abolicionistas (Solana Ruiz y Riopedre 2012), se denuncia de ellos el sensacionalismo con el que se insiste en construcción de la prostituta como víctima tratada y sin agencia (Oso 2000) y se les critica por invisibilizar la prostitución que se ejerce de manera voluntaria, así como la magnificación del engaño de las mafias y redes a las mujeres que llegan a España a prostituirse. Desde esta posición, se valoran de modo negativo los intentos que, desde el Gobierno español, se realizaron para la desaparición de los anuncios de sexo de pago de la prensa, pues se considera que la prostitución de piso (aquella que se anuncia en la prensa) es precisamente la que se ejerce en condiciones menos precarias y más autónomas.

\section{Metodología}

La investigación que da origen a este artículo está basada en la combinación de las técnicas de análisis de contenido - en concreto, el análisis hemerográfico diacrónico diseñado por Amparo Moreno (1998) ${ }^{10}$ y de análisis del discurso, con base en las teorías de van Dijk (1990). Optamos, por lo tanto, por una estrategia metodológica que se asienta en los métodos mixtos de investigación. De esta forma, con su uso conjunto, quisimos sumar las fortalezas del análisis del conte-

9 Es preciso diferenciar la prostitución de la trata y el tráfico de mujeres con fines de explotación sexual. Según la definición de la ONU, adoptada en el Plan Integral de Lucha contra la trata de seres humanos con fines de explotación sexual, aprobado en 2008 en España, trata (en inglés, trafficking in human beings) es el «reclutamiento, transporte, embarque o recepción de personas, por medio de amenaza, uso de la fuerza, coacción, fraude, engaño, abuso de poder o de situaciones de vulnerabilidad, o mediando pago o beneficio económico en la obtención del consentimiento de una persona para que ceda el control sobre otra con el propósito de su explotación. La explotación incluye, como mínimo, la derivada de la prostitución y de otras formas de explotación sexual, trabajos o servicios forzados, esclavitud o prácticas similares, servidumbre y extracción de órganos». El tráfico (en inglés, smuggling of migrants) se entiende, también según la ONU, como «el procedimiento para obtener, directa o indirectamente un beneficio económico de entrada ilegal de una persona en un país del que no es nacional ni residente permanente». Nos inspiramos en la variante de análisis de contenido propuesta por Moreno por dos motivos clave. Por un lado, la propuesta metodológica pone el foco en las personas que protagonizan la información, a través de la respuesta a cinco preguntas claves: ¿Quién enfoca? ¿A qué protagonistas? ¿Haciendo qué? ¿En qué escenarios? ¿Con qué fuentes? Esto le permite constatar la presencia mayoritaria en la prensa de lo que denomina como «arquetipo viril masculino» (Moreno 1998, 32). Por otro lado, el hecho de que los datos sean recogidos en su literalidad (es decir, se transcriben tal y como son mencionados en la unidad redaccional analizada) facilita, además, una lectura cualitativa. Pongamos un ejemplo: no sólo contabilizamos el número de mujeres prostitutas que protagonizan la información sino que, además, recogemos cómo el medio las nombra y las describe, así como los actos y las palabras que se les atribuyen. 
nido y del análisis del discurso y contrarrestar sus debilidades. ${ }^{11}$ Del análisis de contenido obtuvimos datos cuantitativamente representativos que del análisis del discurso en sí no podríamos conseguir. Por su parte, el análisis del discurso hizo posible llegar a una profundización en el contenido, y en el discurso e ideología ocultos tras ese contenido, que no nos permitía el análisis de contenido. Fue, precisamente, el análisis del discurso el que nos abrió el camino para el estudio en profundidad de la construcción mediática del estigma.

Los datos y reflexiones que, a continuación, se aportan, son resultado de un trabajo de campo en el que se analizaron todos los contenidos periodísticos - informativos, interpretativos y de opinión -, con la prostitución como tema central, publicados por El País y ABC en los años 1977, 1987, 1997, 2007 y 2012. Nos interesaba analizar la evolución del tratamiento de la prostitución desde el final de la dictadura franquista. Es por ello que escogimos el año 1977, en los inicios de la Transición democrática española y, a partir de ahí, decidimos analizar un año por década hasta el momento en que finalizamos esta investigación, el 2012. Fueron en total, casi un millar las unidades redaccionales estudiadas. En concreto, 957, de las cuales 544 fueron publicadas por ABC y 413 en El País. A todas ellas se les aplicó, conjuntamente, el análisis de contenido y el análisis del discurso.

\section{La construcción del estigma en la prensa española: El País y ABC}

El País y el $A B C$ siguen caminos distintos para la construcción del estigma, pero coinciden: a) en un punto de partida (el considerar a la mujer que se prostituye antes prostituta que mujer o persona, en tanto que esa es la condición de ella que se destaca en primer lugar), y b) una confluencia final, evidenciada en los últimos años analizados: la construcción mediática del estigma de la «víctima perfecta».

El estigma se pega a la piel, como lo hacían, en la antigua Grecia, las marcas con las que se grababan a los esclavos y esclavas, y de las que procede la palabra. $\mathrm{Y}$ eso se observa en los medios, y en concreto, en los dos periódicos analizados, cuando se refieren a la persona que se prostituye destacando en primer lugar su condición laboral. Si una mujer es detenida o asesinada, el análisis del discurso realizado nos muestra que es muy probable que sea identificada en los titulares antes como prostituta que como mujer o persona con nombre y apellidos. Así lo muestran ejemplos como los siguientes.

Vicente Mariño (2009) defiende, para las Ciencias de la Comunicación, la integración de estas dos técnicas profusamente empleadas por las y los investigadores de este ámbito disciplinar, aunque casi siempre por separado. Las razones argumentadas por Mariño coinciden en gran medida con los motivos que basaron nuestra propuesta metodológica: la incapacidad del análisis del contenido para abordar el sustrato ideológico que esconde el texto. Sin embargo, mientras que para Mariño el análisis del discurso es un complemento a las debilidades del análisis del contenido, este trabajo parte de la consideración de las dos técnicas al mismo nivel. 
«Detenido el presunto asesino de una prostituta» (El País, 15 de octubre de 1977);

«Una prostituta, detenida en relación con la muerte de un joven sacerdote jesuita» ( $E l$ País, 23 de septiembre de 1987);

«Identifican a una prostituta heroinómana como la decapitada de San Fernando de Henares» (ABC, 28 de agosto de 1987);

«Intenta matar a una prostituta a causa de su impotencia» $(A B C, 1987)$;

«Detenido el presunto asesino de una prostituta que se anunciaba en Internet» ( $E l$ País, 7 de febrero de 1997);

«Arranca el juicio por el asesinato de una prostituta en El Cerro» $(A B C, 20$ de febrero de 2012);

Más allá de ese punto de partida común, la construcción del estigma en $A B C$ y El País, como se ha dicho en un inicio, está determinada por la línea ideológica del medio. Para el conservador $A B C$, la prostitución y las prostitutas serán un mal y una amenaza para un Nosotros identificado con los principios conservadores y católicos. El estigma, desde la perspectiva presente en $A B C$ y de la que El País marca distancias, se construye pues como amenaza a: a) los preceptos morales que consideran a las mujeres y el sexo como tentación y pecado; b) a las y los ciudadanos pertenecientes a clases sociales no desfavorecidas, por la delincuencia que se asocia en el periódico a la prostitución y la disrupción en las normas de convivencia cívica en el espacio público; c) a la salud de las y los ciudadanos que forman parte de ese «Nosotros» con el que el periódico se identifica (la insistencia en la vinculación de la prostitución con enfermedades de transmisión sexual, y más concreto con el SIDA); y d) a la población española por parte de «males» asociados a la población inmigrante.

\section{La prostitución como vicio y comportamiento inmoral}

La estigmatización de la prostituta como vicio y mal moral está presente fundamentalmente en el discurso del periódico conservador (no en el de El País) de las décadas de los 1970 y 1980. Su identificación como tal llega a ser explícita en el propio discurso de la persona que elabora la información:

El tema que hoy trata el magistrado juez de Peligrosidad Social, don Manuel Rico Lara, es el de la prostitución, así como el de la actuación, condenada por todos, de los que explotan este vicio («La prostitución está ante nosotros», en $A B C$ del 21 de mayo de 1977).

La prostitución es identificada con anormalidad. Así ocurre en una de las cartas al director publicada en 1987. «Lo anormal seguirá siendo siempre anormal», dice la lectora, quien considera que las prostitutas están siendo un modelo para la juventud, lo que de fondo encierra la estigmatización también de la idea de libertad sexual y la identificación como «putas» de las mujeres que viven libremente su sexualidad. 
Me ha llamado mucho la atención el programa de TV «En familia». En él se trató esta vez el tema de la prostitución. Me hizo reflexionar sobre esta realidad tan vieja como la vida misma. Estas señoras hablaron desde su frustración ellas que son lo que hoy se presenta a la juventud como prototipo de libertad, no expresaban realmente alegría, ni descaro y no lo hacían porque lo anormal seguirá siendo siempre anormal, porque no se puede decir que comerciar con el cuerpo es la felicidad («En familia», $A B C, 1$ de junio de 1987).

El considerar que la prostitución está fuera de la normalidad lleva a defender la «reinserción social» como solución para recuperar dicha normalidad:

Askabide, camino de libertad, es el nombre que un grupo de religiosas ha dado al centro de acogida creado por ellas para acoger a las prostitutas bilbaínas que quieran intentar reinsertarse normalmente en la sociedad («Religiosas abren un centro de acogida para prostitutas», publicada en $A B C$ el 3 de agosto de 1987).

El País, que nace en 1976, recién finalizada la dictadura franquista, se aleja de este estigma. No lo encontramos en sus páginas. Al contrario, introduce, tanto en sus contenidos informativos como en los de opinión, un discurso que condena la doble moral de la sociedad española respecto a la prostitución y da entrada a la voz de las prostitutas y a la defensa de sus derechos. En sus contenidos de opinión, condena la hipocresía de una sociedad que estigmatiza a la prostituta y censura la libertad sexual aun siendo la prostitución una práctica de consumo masculino generalizada. Porque, tal y como expresa en el periódico una de sus más prestigiosas firmas, Paco Umbral, «un sistema cerradamente puritano necesita la cloaca de la prostitución como la belleza de Venecia necesita la podredumbre de sus canales» («Las respetuosas», El País, 12 de marzo de 1977). En su discurso informativo, les dará voz a las prostitutas cuando se manifiestan frente a la Ley de Peligrosidad Social, aprobada en las postrimerías del franquismo, que hace que sobre ellas penda permanentemente la amenaza de la cárcel. Un ejemplo claro es esta información sobre una manifestación de prostitutas bilbaínas, en noviembre de 1977, que no será cubierta por el $A B C$.

Más de cincuenta prostitutas se encerraron ayer por la tarde en un pabellón del Hospital Civil de la capital vizcaína para pedir la abolición de la ley de Peligrosidad Social y exigir que se aclaren las circunstancias que rodearon la muerte de su compañera María Isabel Gutiérrez, que se prendió fuego a lo bonzo, la noche del pasado martes, en su celda de la cárcel de Basauri (Vizcaya) («Manifestación de prostitutas bilbaínas contra la ley de Peligrosidad Social», El País, 12 de noviembre de 1977).

Las divergencias con $A B C$ en lo que respecta a moral sexual son notorias y se ponen de manifiesto cuando, en 1997, el gobierno del PP incorpora de nuevo el delito de corrupción de menores en el Código Penal, que había sido eliminado en 
su reforma del año 1995, en los últimos meses de la etapa del PSOE en el poder, con Felipe González al frente. Mientras en $A B C$ se defiende la medida, El País la critica, argumentando que ya existen instrumentos normativos para castigar los abusos sexuales a menores y que la recuperación de tal tipo penal podría servir de excusa jurídica para censurar la libertad sexual. Así se pronuncia en uno de sus editoriales:

La corrupción de menores, un delito indefinido que se ha utilizado en ocasiones para sancionar prácticas sexuales que no atentaban contra la libertad sexual, no es el mejor mecanismo para sancionar las conductas de unos adultos que, si se confirman los indicios, han abusado sexualmente de niños de 12 años o menos, y los han prostituido y utilizado para producir pornografía (editorial «Corrupción de mayores», El País, 1 de agosto de 1997).

Las diferencias entre ambos se seguirán manifestando, también en años posteriores, en la cobertura de los conflictos vecinales con la prostitución de barrio. $A B C$, posicionado del lado de las voces vecinales, recogerá los argumentos de los vecinos relativos a la moral y el incivismo de las prácticas sexuales en la calle. El País no insistirá en dichos argumentos y contrarrestará las voces de las vecinas y vecinos de los barrios afectados con el testimonio de las personas que allí se prostituyen.

En los últimos años, como ya hemos adelantado, estas aristas se liman para construir la confluencia de ambos periódicos alrededor del discurso abolicionista y de la victimización de las mujeres que ejercen la prostitución. Esta confluencia tiene su correlato con la que se produce, en el ámbito político, entre el discurso de derechas y de izquierdas. El discurso de derechas alrededor de la prostitución y su condena desde una perspectiva moral acabará encontrándose en parte con el discurso de izquierdas que promueve la abolición de la prostitución por considerarla una manifestación más del sexismo y de la violencia contra las mujeres.

\section{La prostitución como delincuencia y disrupción de la convivencia cívica}

Este perfil estigmatizador está muy en relación con una de las constantes de la representación de la prostitución en la prensa española, su tratamiento como suceso. El abordaje como suceso está presente, en $A B C$, en el $34 \%$ de los contenidos analizados, y en el 34\% en El País. La representación de la prostituta como delincuente se asocia no sólo a su criminalización como tal prostituta sino también a la criminalización que se hace en general de la población que subsiste en los márgenes de la sociedad, tal es el caso de la inmigración y de las personas afectadas por drogodependencias o enfermedades como el SIDA.

Al centro le puede el cáncer de la desesperanza. Prostitutas, travestidos, carteristas, tironeros, «cogoteros», asaltadores, traficantes, chulos gamberros, vándalos, «inmigrantes-ilegales-profesionales-del-delito». Y miedo [...] Con la Ley en la mano, 
la Policía no puede hacer nada para evitar la prostitución y las fulanas ni se inmutan ante su presencia. Todos señalan al submundo que se mueve en torno a este tráfico como el gran mal. Chulos, como el que aparece en esta imagen con «su» prostituta, sobre estas líneas reparten heroína y cocaína entre las protegidas («La delincuencia mata la esperanza en el centro», en $A B C$ del 19 de diciembre de 1997).

La presencia de las personas que ejercen la prostitución como fuente de conflicto con las y los otros vecinos del barrio será una constante en el $A B C$ a lo largo del período analizado. En dicho conflicto, el $A B C$ se sitúa sistemáticamente del lado del vecindario, a quienes con frecuencia da voz, frente a las prostitutas, de las que desconocemos su experiencia, su testimonio y su mirada. Lo dicho se comprueba en esta investigación no solo mediante el análisis cualitativo del discurso sino también a través de los datos cuantitativos obtenidos del análisis de contenido. El marco interpretativo asociado a la prostitución como fuente de conflicto en la convivencia ciudadana está presente en el 23,4\% de los contenidos en el $A B C$ y se reduce a más de la mitad en El País, que presenta dicho marco interpretativo en el $10,4 \%$ de las unidades redaccionales analizadas, y se diferencia del $A B C$ en que nos permite saber cuál es el punto de vista de las personas que se prostituyen.

En cuanto al discurso, mientras en $A B C$ el vecindario aparece asociado a verbos que muestran su acción en positivo y a actos de habla, todo lo contrario ocurre con quienes ejercen la prostitución: «Los vecinos de Marconi logran que las prostitutas dejen la zona de viviendas» $(A B C, 6$ de septiembre de 2007), «Los vecinos de Marconi se mostraron satisfechos por el resultado de la reunión» $(A B C, 7$ de septiembre de 2007), «La barriada Paz y Amistad, cercada por grupos de gamberros y por prostitutas» $(A B C, 16$ de junio de 2007).

Observamos, en el discurso mediático, una contradicción no menor, sobre todo en los últimos años de la muestra, en los que se extiende el discurso de la trata de mujeres. $\mathrm{Y}$ esta contradicción se reflejará tanto en los contenidos informativos de El País como en el $A B C$. Por una parte, se las trata como víctimas de las mafias. Por otra, se las criminaliza al presentarlas como elemento disruptor de la convivencia cívica en la calle y fuente de tensión con el vecindario de los barrios en los que ejercen.

Esta contradicción entre la victimización y, al mismo tiempo, la criminalización de la prostituta llega a manifestarse incluso en discursos divergentes entre lo que el texto de la noticia dice sobre la prostituta y el modo en el que la fotografía la representa, lo que podemos ver en este ejemplo extraído del $A B C$. El texto representa a las jóvenes como víctimas. La imagen las muestra según parámetros icónicos propios de la representación del criminal que huye de su identificación. Sus rostros aparecen ocultos, como si fuesen criminales protegiendo su identidad. 


\section{Imagen 1}

Ejemplo de criminalización en la imagen

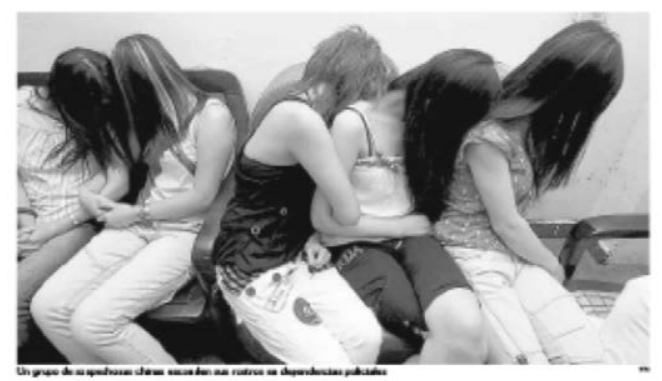

\section{Seis mafias se repartían los 14 millones del negocio de la prostitución china:}

Fuente: «Seis mafias se repartían los 14 millones del negocio de la prostitución china», ABC, 23 de febrero de 2007

Este discurso, del que la prensa se hace eco, tiene su origen en el discurso político español vigente en ese momento y que se extiende hasta la actualidad. Existe una contraposición entre la legislación y las políticas estatales y las normativas autonómicas y locales. El Código Penal español castiga a quien se beneficia de la prostitución ajena, aunque ésta sea consentida, pero no a quien se prostituye. Así mismo, las políticas estatales, con marco en el Plan Integral de Lucha contra la Trata de Mujeres y Niñas con Fines de Explotación Sexual, inciden en la prostitución como mayoritariamente forzada y en la prostituta como víctima de las redes de trata y tráfico, y contemplan medidas para que puedan denunciar a las mafias que las explotan. Sin embargo, las políticas locales que se pusieron en marcha en varios ayuntamientos y comunidades autónomas, como Cataluña, van en sentido contrario y, en ciertos casos, llegan a multar a quien se prostituye y/o a quien compra servicios sexuales en la calle.

La prostitución como amenaza a la salud

Ya habíamos visto en O’Neill et al. (2008) como, a la construcción del estigma, contribuye la vinculación de la prostitución a las enfermedades de transmisión sexual. Esta relación fue muy fuerte en el discurso mediático de la década de los 1980, en plena crisis del SIDA. Los datos cuantitativos así nos lo muestran. Tanto en $A B C$ como en El País, 1987 es el año en que publican más contenidos en los que se relaciona prostitución con problemas de salud. Llegan a suponer hasta el 10,2\% de los analizados ese año en $A B C$, y el 14\% en El País. Nunca, ni en los años anteriormente estudiados, ni en los posteriores, este vínculo fue tan fuerte.

No obstante, una cosa es la información sobre prostitución y enfermedades de transmisión sexual, básicamente el VIH, y otra es la construcción del estigma. Este estigma está muy presente en $A B C$, en el año 1987, en plena crisis del SIDA, pero no en El País, que adopta una línea editorial por completo contraria. Mien- 
tras el $A B C$ insiste en informaciones en las que la persona que se prostituye es condenada como amenaza social por ser considerada agente transmisor de SIDA, El País intenta desmontar este estigma e incluye contenidos en los que se pone de manifiesto que no solo la persona que se prostituye, sino también, el cliente, es responsable.

En $A B C$, la prostituta, sobre todo si es mujer transgénero, es considerada una amenaza para la sociedad por su potencial de contagio (por ejemplo, en informaciones como «La prostitución de travestidos, un peligro sanitario, según los vecinos de Vitruvio. En la zona existen un instituto y varias guarderías», $A B C, 27$ de febrero de 1987). No se alude en ningún momento a base científica alguna para que el VIH se ligue sobre todo a las, en aquel momento, etiquetadas como «travestis», de lo que se deduce una estigmatización implícita de toda sexualidad no heteronormativa basada en la construcción del mito. Es decir, en el alejamiento entre la realidad y el discurso mediático que se construye. Nada se dice, en todo caso, de la responsabilidad de los compradores de sexo en la transmisión de enfermedades venéreas.

El discurso político parece ir, en ese momento, por detrás del discurso mediático. Ante la alarma social a la que contribuyen los medios, se anuncian medidas políticas desde partidos e instituciones gubernamentales con propuestas como el confinar a las personas que ejercen la prostitución en zonas específicas.

La persona que se prostituye y ha sido contagiada por VIH es, incluso, animalizada en los contenidos: «Una prostituta con SIDA podría estar suelta en la ciudad, ya que se marchó del hospital después de haberse realizado el diagnóstico» («Veinte extranjeros, expulsados de la República Federal de Alemania por tener el virus del SIDA, $A B C, 29$ de marzo de 1987). Puede, además, ser presentada como un ser macabro y malintencionado: «Cadena perpetua a un enfermo de SIDA que estranguló a tres compañeros de orgía. Se vengaba del mal dando muerte a sus clientes homosexuales» ( $A B C, 12$ de julio de 1987).

El periódico traslada y amplía la alarma social por la transmisión del VIH no sólo a su discurso informativo, sino también a contenidos de opinión de carácter editorializante, tal es el caso de ZigZag, que incluye pequeños textos de opinión sin firma (sueltos) que muestran la línea editorial del periódico:

A mediados de febrero, representantes de la Asociación Popular de Vecinos Altos del Hipódromo de Madrid fueron recibidos por el doctor Nájera. Estos padres de familia le expusieron la preocupación por el contagio de SIDA y hepatitis B que corre la población infantil en la zona de la calle Vitruvio, debido al posible contagio infantil con jeringuillas, preservativos y otros detritus que habitualmente deja la prostitución de travestidos en aquella zona y en el interior de determinados colegios allí ubicados. Después de escucharlos atentamente, el doctor Nájera fue breve y lacónico en su respuesta: «Los niños - argumentó - no corren ningún peligro, ya que no son tan tontos como para coger y jugar con jeringuillas del suelo.» Recientemente - y esto convendría que lo supiese el doctor Nájera -, en el colegio público Maestro José Regidor, de Huelva, 
varios niños se contagiaron de hepatitis B por jugar, precisamente, con jeringuillas usadas que se habían encontrado en la calle ( $A B C$, «Rafael Nájera», 17 de abril de 1987).

El País muestra, en el mismo año, un discurso por completo opuesto. Se alude a las responsabilidades de los compradores de sexo en la transmisión del SIDA, se defiende a las prostitutas transgénero frente a su estigmatización y se adopta el discurso de la defensa de los derechos de las mujeres que realizan trabajo sexual, lo que se muestra, por ejemplo, en la entrevista que realizan a Pía Covre como representante de las prostitutas italianas (había fundado, en 1982, el Comitato per i diritti civili delle prostitute). El periódico da, así mismo, amplia cobertura a las jornadas sobre prostitución que promueve ese año el ayuntamiento de Madrid, gobernado por el PSOE, y que en el $A B C$ llegan a ser ridiculizadas. Los ejemplos siguientes evidencian la distancia entre los discursos opuestos de $A B C$ y de El País en la cobertura de dichas jornadas y, más en concreto, en la postura que en ellas se adopta acerca de la importancia del compromiso del cliente en el uso del preservativo para evitar la extensión del VIH.

«Las prostitutas», dijo, «deberían exigir el uso de preservativos a los clientes, puesto que ellas, al menos las que no se drogan, no tienen la enfermedad» (palabras de Pilar Estébanez, directora del Centro de Promoción de la Salud del distrito de Centro (CPS) a propósito de las jornadas sobre prostitución. «Mejorar la situación de las prostitutas, objetivo del debate que se inicia hoy», El País, 7 de abril de 1987). ${ }^{12}$

Sobre este asunto de la prostitución, en este caso femenina, parece que se había dicho casi todo; pero no es así. Ahora de lo que se trata es de realizar una campaña de solidaridad, según la doctora Pilar Estébanez [...]. «Que no se quede una prostituta sin usuario porque no lleve preservativo», dijo («Piden solidaridad para que los clientes de las prostitutas usen preservativos. Juez Navarro: «Piden que dar la mano a estas hermanas»», $A B C, 4$ de agosto de 1987).

El País mantiene una postura activa en la desestigmatización de quien ejerce la prostitución como transmisor del VIH, hasta el punto de afirmar en un editorial, en el que se manifiesta en contra de las propuestas de recluir a las prostitutas en guetos, que «la presencia de portadores de anticuerpos del SIDA - si lo son - en las calles no representa ningún riesgo» («Las prostitutas condenadas», El País, 14 de agosto de 1987). La afirmación será puesta en cuestión en la carta al director escrita días después por un médico adjunto de medicina interna.

En ambos casos, pues, tanto el afán estigmatizador como desestigmatizador, en relación con la persona que se prostituye como agente de transmisión de VIH, lleva a informaciones alejadas de la realidad.

12 Disponible en http://elpais.com/diario/1987/04/07/madrid/544793063_850215.html 


\section{La inmigración como amenaza a la población nacional}

La prostitución ejercida por personas inmigrantes se estigmatiza como amenaza para el «Nosotros» como colectivo nacional español, y se hace siguiendo un esquema triangular en las representaciones mediáticas: 1) el hombre inmigrante vinculado a la delincuencia; 2) la mujer inmigrante que ejerce la prostitución y víctima del hombre inmigrante como proxeneta o parte de redes de trata, y 3) la policía, que actúa de salvadora de la mujer inmigrante explotada y tratada. En los últimos años analizados se incorpora, en los dos medios, el uso del verbo «liberar» para referirse a la acción de la Policía.

Liberada en Almería una joven secuestrada en Rumanía y obligada a ejercer la prostitución (El País, 15 de julio de 2007).

Liberación de mujeres. El objetivo, según Amato, es «combatir los grupos criminales que organizan el tráfico y devolver la libertad a estas muchachas» («Más de 700 detenidos en Italia por tráfico de personas y prostitución», $A B C, 25$ de enero de 2007). La representación mediática ofrece así una imagen en blancos y negros que no tiene en cuenta los matices. Las operaciones policiales pueden no ser una «liberación» para aquellas mujeres que se encuentran en el país en situación administrativa irregular y/o que han normalizado la explotación en la que viven y, en cierto modo, han construido en ella su círculo de seguridad en un país que desconocen.

Este triángulo se consolida a lo largo del período analizado tanto en $A B C$ como en El País y cristaliza, en los últimos años objeto de estudio, en la creación de un nuevo estigma: el de la «víctima perfecta», del que hablaremos de manera más pormenorizada en el siguiente apartado.

No sólo a través de la victimización se produce la objetualización de la prostituta inmigrante (como objeto de la acción de otros, que no tiene agencia, es decir que no tiene capacidad de actuación) sino también por medio de otra estrategia, en este caso gramatical: la substantivación del gentilicio, lo que, de manera subyacente, lleva a considerarlas antes inmigrantes que personas, y produce en el lector o lectora una sensación de alejamiento que obstaculiza la empatía. Tal ocurre en los siguientes ejemplos, que son sólo una pequeña muestra de las varias expresiones de construcción similar localizadas a lo largo del período analizado:

El motivo ha radicado en la competencia desleal que, a juicio de las españolas, practican las portuguesas («Prostitutas madrileñas protestan por competencia desleal de las portuguesas», El País, 1977)

Las búlgaras, que se alojaban en un piso de la calle de Camarena, entregaban todo el dinero que recaudaban («Detenida una red que forzaba a mujeres búlgaras a prostituirse», El País, 1 de junio de 1997). 


\section{La prostituta como la víctima perfecta}

Sin embargo, las divergencias alrededor de la construcción del estigma menguarán en los últimos años analizados. Ambos medios acabarán por coincidir en representar a la prostitución fundamentalmente como forzada, dejando a un margen la de carácter voluntario. En $A B C$, los estigmas que identifican a la mujer que se prostituye como amenaza se suavizan: la prostituta deja de ser representada fundamentalmente como amenaza para ser presentada como víctima. No hay términos medios. La única forma de no ser una «mala mujer» trabajando en la prostitución es que te fuercen a ejercerla.

Alrededor de las narrativas de la trata de personas con fines de explotación sexual, se extiende el estigma de la «víctima perfecta». Pérez Freire y Casado Neira identifican la «víctima perfecta» con «la hipervíctima tratada, mujer vulnerable y vulnerada en sus derechos humanos de forma extrema (violentada) y rescatada por parte de la sociedad personificada preferentemente por las fuerzas y cuerpos de seguridad del estado (Guardia Civil y/o policía)» (Pérez Freire y Casado Neira 2015, 35). El discurso de la «víctima perfecta» se extiende con el asentamiento en la prensa española del discurso abolicionista hegemónico en el discurso político vigente en ese momento y aún ahora, en el momento de elaboración de este artículo, y en el que coinciden los dos partidos políticos mayoritarios. Este discurso se basa, precisamente, en la narrativa de la trata de mujeres y en la explotación forzada.

Tanto el análisis cualitativo como el cuantitativo muestran la confluencia de ambos medios en el estigma de la «víctima perfecta» en los últimos años analizados. En el caso de $A B C$, esta construcción, localizada en el $11 \%$ de las unidades redaccionales analizadas en 1977, se incrementa hasta el 32\% en el último año observado, el 2012. En El País, el estigma de la víctima perfecta pasa de estar presente en un 5,2\% en el 1977 a un $42 \%$ en el 2012. El dato es coherente con la elevada presencia de informaciones sobre trata de seres humanos con fines de explotación sexual en los últimos años de la muestra. En ambos periódicos, la trata apenas es abordada en las décadas de los 1970 y 1980. Sin embargo, su presencia se incrementa notablemente a partir de la década de los 1990 y sobre todo con la entrada del nuevo milenio, coincidiendo con la globalización de los flujos de la prostitución (del sur empobrecido al norte rico) y la conversión de España en país de recepción de prostitución. En el caso de $A B C$, los contenidos sobre trata llegan al 9\% en 2007 y al 18\% en 2012. En cuanto a El País, supone un 19\% en 2007 y un $12 \%$ en 2012.

La construcción del estigma de la «víctima perfecta» se refuerza cuando va acompañado del estereotipo de «madre sufriente», como ocurre con la cobertura de la lucha de la considerada «madre coraje», la argentina Susana Trimarco, tanto por localizar a su hija, Marita Verón, secuestrada y prostituida, como por hacer justicia con sus captores. O cuando, en el abordaje del juicio a Ioan Camplaru, conocido como «Cabeza de Cerdo» y reconocido mediáticamente como uno de los mayores capos en la trata de seres humanos con fines de explotación sexual, 
alguna de sus víctimas narra cómo fue obligada a abortar («Yo no quería abortar pero Ioan me obligó a hacerlo» (El País, 1 de febrero de 2012).

Argentina, una vez más, está conmocionada. María de los Ángeles Verón, más conocida como Marita, fue secuestrada, esclavizada y obligada a prostituirse hace diez años. Desde entonces, su madre, Susana Trimarco, la busca y demanda justicia pero no logra ninguna de las dos cosas («Sin justicia para la madre coraje argentina», $A B C$, 14 de diciembre de 2012)

Susana Trimarco reunió a sus 47 años el arrojo suficiente y buscar a su hija María de los Ángeles, Marita, Verón en los prostíbulos de Argentina. Cruzando testimonios por aquí y por allá fue informándose de que la chica había sido golpeada con la culata de una pistola en las calles de San Miguel de Tucumán, introducida en un coche rojo, vendida a una red de trata de blancas, drogada, violada, apuñalada y forzada a tener un hijo en cautiverio con su proxeneta, José Fernando Gómez, alias el Chenga. («Susana Trimarco, 'madre coraje argentina': «Así como yo no tengo paz, tampoco ellos la van a tener» (El País, 18 de marzo de 2012) $)^{13}$

Es decir, la prostituta se representa como víctima sin ambages, sin tener en cuenta que la realidad de la prostitución está llena de grises, que no toda la prostitución es forzada, que no todas las prostitutas se consideran a sí mismas víctimas y que, aún en casos de trata, puede haber un grado de agencia y capacidad de decisión de las mujeres, como ocurre cuando son engañadas sólo en parte: saben que su destino en España es la prostitución pero ignoran las condiciones de precariedad, violencia o incluso vulneración de los derechos humanos y laborales en las que van a trabajar. O cuando, a pesar de las condiciones de explotación, encuentran en esas rejas un cierto espacio de seguridad, en el que tejen sus relaciones personales y afectivas y en el que encuentran un sustento económico, en un país que desconocen y que no les ofrece mayores alternativas.

En esta evolución hacia el estigma de la víctima perfecta, el $A B C$ llega a cambiar de modo radical su discurso acerca de la prostitución de calle y las protestas que ésta genera en el vecindario de las zonas en las que se ejerce. A lo largo de todo el período analizado, excepto en el 2012, el periódico se pone sistemáticamente del lado del vecindario y del argumentario por él utilizado (inseguridad, inmoralidad, mala imagen...), ofreciendo así una representación culpabilizadora y criminalizadora de la prostituta, a la que acostumbra no darle voz. Sin embargo, en el último año analizado, aunque $A B C$ sigue defendiendo la eliminación de la prostitución en la calle, los argumentos que recoge son totalmente distintos: es preciso acabar con la prostitución en la calle para luchar contra la explotación sexual de las mujeres. De este modo, el estigma de la «víctima perfecta» se usa para reforzar los argumentos a favor

13 Disponible en http://sociedad.elpais.com/sociedad/2012/02/16/actualidad/1329419719 938148. $\underline{\mathrm{html}}$ 
de la desaparición de las prostitutas del espacio público, en una actitud paternalista, según la cual se decide por ellas lo que es mejor para ellas pero sin consultarlas.

\section{Conclusiones}

El estigma se refuerza en el desconocimiento del «Otro», en este caso, de la «Otra». En una sociedad en la que el acceso a la realidad está ampliamente mediatizado, el estigma que rodea a la prostitución, y que se expande en la intersección de las discriminaciones de género, étnicas, nacionales, de orientación sexual y de clase social, encuentra aliado y refuerzo en los contenidos difundidos por la prensa y por los medios de comunicación en general, tal y como nos permiten concluir los datos de esta investigación.

En $A B C$ y en El País, la construcción del estigma es divergente, porque el «Nosotros» del que parten es distinto y está definido por líneas ideológicas diferentes y, sólo hasta cierto punto, opuestas. El $A B C$ traslada a la prostitución los miedos y preconceptos que nacen del ideario conservador y católico del que bebe. En $A B C$, la mujer que se prostituye es una amenaza para los principios morales del catolicismo, y se concibe así mismo como amenaza para la propia integridad (identitaria, nacional, sanitaria, física y de la propiedad). El País, sin embargo, se aleja conscientemente de esta postura conservadora y moralista en lo sexual.

No obstante, aunque ese «Nosotros» del que parten ambos periódicos es divergente tiene una característica común: la mirada heteropatriarcal. Para ambos, la mujer que se prostituye es la «Otra», sea ésta más o menos estereotipada, y es, por lo tanto, antes prostituta que mujer o persona. En ambos está presente una mirada simplificadora que impide ver todas las aristas que rodean a la realidad de la prostitución. Esa simplificación, unida al predominio del discurso abolicionista en la política española de la última década (presente en los dos partidos mayoritarios en el período analizado, el PP y el PSOE), hace que ambos medios confluyan en el estigma de la víctima perfecta.

\section{Referencias bibliográficas}

Alba, Yolanda. 2006. «La prostitución en los mass-media hoy». Crítica 940: 52-56.

Andrade, Leandro Feitosa. 2004. Prostituição infanto-juvenil na mídia: estigmatização e ideologia. São Paulo: EDUC-Fapesp.

Barthes, Roland. 1957. Mitologías. Madrid: Siglo XXI. Disponible en el enlace: http://www. ddooss.org/libros/mitologias_Roland_Barthes.pdf [Consultado en 8 de marzo de 2017].

Benoit, Cecilia, y Helga Hallgrimsdóttir, Helga. 2011. Valuing Care Work: Comparative Perspectives. Toronto: University of Toronto Press.

Brufao, Pedro. 2011. Las miserias del sexo. Prostitucion y políticas públicas. Madrid: La Catarata. Caruncho, Cristina. 2010. «Ética e feminismo». Pp. 13-23 in Claves para unha información non 
sexista, organizado por Luís Álvarez Pousa y Belén Puñal. Santiago de Compostela: Atlántica.

Cortes Generales del Estado (2007). «Informe de la ponencia sobre la prostitución en nuestro país. Aprobada en sesión de la ponencia de 13 de marzo de 2007». Madrid: Boletín Oficial de las Cortes Generales.

El País. 2014, 25 de septiembre. «El INE estima que prostitución y narcotráfico elevan el PIB un 0,85\%». Disponible en el enlace http://economia.elpais.com/economia/2014/09/25/ actualidad/1411634 [Consultado en 20 de abril de 2017]

Fong, Ting, Eleanor Anne Holroyd, y William C.W. Wong. 2013. «Dangerous women of Hong Kong? Media construction of stigma in female sex workers». Journal of Behavioral Health 2 (1): 59-65. Doi: https://doi.org/10.5455/jbh.20120607063411

Gallego Ayala, Juana. 2010. «Produción informativa e mecanismos de reprodución de estereotipos de xénero nos medios de comunicación». Pp. 32-33 in Claves para unha información non sexista, organizado por Luís Álvarez Pousa y Belén Puñal. Santiago de Compostela: Atlántica.

Goffman, Erving. 2006. Estigma. La identidad deteriorada. Buenos Aires: Amorrortu. Originalmente publicado en Erving Goffman, Stigma. Notes on the management on spoiled identitity (Prentice Hall, 1963)

Iglesias, Juan et. al. (2015). Estudio sobre la situación laboral de la mujer inmigrante en España. Organización Internacional para las Migraciones. Disponible en el enlace http://www. spain.iom.int/sites/default/files/Estudio \%20Sobre\%20la\%20Situaci\%C3\%B3n\%20 Laboral\%20de\%20la\%20Mujer\%20Inmigrante.\%20OIM\%202015.pdf [Consultado el 16 de abril de 2017)

Janzen, Caitlin, Susan Strega, Leslie Brown, Jeannie Morgan, y Jeannine Carrière. 2013. «Nothing Short of a Horror Show: Triggering Abjection of Street Workers in Western Canadian Newspapers». Hypatia, 28 (1): 142-162. Doi: https://doi.org/10.1111/j.1527$-2001.2011 .01256 . x$

Juliano, Dolores. 2005. «El trabajo sexual en la mira. Polémicas y estereotipos». Cadernos Pagu, 25: 79-106. Doi: https://doi.org/10.1590/S0104-83332005000200004

Mariño, Vicente. 2009. Desde el análisis de contenido hacia el análisis del discurso. La necesidad de una apuesta decidida por la triangulación metodológica. Disponible en el enlace http://uvaes.academia.edu/MiguelVicenteMarino/Papers/202303/Desde_el_analisis_ de_contenido_hacia_el_analisis_del_discurso._La_necesidad_de_una_apuesta_decidida_por_la_triangulacion_metodologica [Consultado en 8 de marzo de 2017].

Ministerio de Sanidad, Servicios Sociales e Igualdad. (2015). II Plan Integral de Lucha contra la Trata de Mujeres y Niñas con Fines de Explotación Sexual (2015-2018). Disponible en el enlace http://www.msssi.gob.es/va/ssi/violenciaGenero/tratadeMujeres/planIntegral/DOC/Plan_Integral_Trata_18_Septiembre2015_2018.pdf [Consultado en 23 de diciembre de 2016].

Moreno Sardá, Amparo. 1998. La mirada informativa. Bosch: Barcelona.

Oliveira, Alexandra. 2011. Andar na vida: prostituição de rua e reacção social. Coimbra: Almedina.

O'Neill, Maggie, Rosie Campbell, Phil Hubbard, Jane Pitcher, JaneScoular. 2008. «Living with the Other: Street sex work, contingent communities and degrees of tolerance». Crime Media Culture 4 (1): 73-93. Doi: https://doi.org/10.1177/1741659007087274

Oselin, Sharon S. 2009. «Leaving the streets: transformation of prostitute identity within the prostitution rehabilitation program». Deviant Behavior 30(4): 379-406. https://doi. org/10.1080/01639620802258485 
Oso, Laura. 2004. «Mulleres inmigrantes latinoamericanas e traballo sexual en Galicia. Pp. 23-57 in Outras voces, outros mundos. Mulleres inmigrantes e prostitución en Galicia», VV.AA. Santiago de Compostela: Andaina-Concello de Santiago, Concellería de Emigración e Inmigración.

Oso, Laura. 2000. «Estrategias migratorias de las mujeres ecuatorianas y colombianas en situación irregular: servicio doméstico y prostitución en Galicia, Madrid y Pamplona». Comunicación presentada al II Congreso sobre la migración en España. Madrid, 5-7 de octubre.

Pérez Freire, Silvia, y Casado Neira, David (2015). «Sexo, masculinidades y las víctimas expiatorias. Las mujeres en contexto de prostitución en los medios: La mirada desde las ONG». RICD 1 (2). Disponible en el enlace http://www.usc.es/revistas/index.php/ ricd/article/view/2650/2816 [Consultado en 10 de marzo de 2017]

Pérez Wolfram, Clara (2003). «Las inmigrantes en la prensa: víctimas sin proyecto migratorio». Mugak 24. Disponible en el enlace http://www.pensamientocritico.org/claper1103. htm [Consultado en 16 de abril de 2017)

Pitman, Beverley A. 2002. «Re-mediating the spaces of reality television: America's Most Wanted and the case of Vancouver's missing women». Environment and Planning A 34 (1): 167-184. Disponible en el enlace http://www.envplan.com/abstract.cgi?id=a34134 [Consultado en 10 de marzo de 2016].

Puñal, Ana Belén. 2010. «Entrevista a Laura Seara: 'Os anuncios de contactos non se poden vincular ao dereito á liberdade de expresión'». Tempos Novos 162: 22-23.

Puñal, Ana Belén. 2015. «Presenza e ausencia das mulleres na prensa. Análise do tratamento da prostitución en El País e ABC» (tesis doutoral, Universidade de Santiago de Compostela). Disponible en el enlace http://dspace.usc.es/handle/10347/13376. [Consultado en 26 de diciembre de 2016].

Ribeiro, Manuela et al. 2007. Vidas na raia: prostituição feminina em regiões de fronteira. Porto: Afrontamento.

Rizo, Marta. 2011. «De personas, rituales y máscaras. Erving Goffman y sus aportes a la comunicación interpersonal». Quórum académico 8 (15): 78-94. Disponible en el enlace https://dialnet.unirioja.es/descarga/articulo/3998939.pdf [Consultado en 10 de marzo de 2017].

Rosemberg, Fúlvia, y Leandro Feitosa Andrade. 1999. «Ruthless rhetoric - Child and youth prostitution in Brazil». Childhood - A global Journal of Child Research, 6 (1): 113-131. Doi: doi.org/10.1177/0907568299006001009

Sacramento, Octávio, y Manuela Ribeiro. 2014. «Mulheres marcadas: prostituição, ordem e exclusão». Cuadernos de trabajo social 27 (1): 197-209. Doi: https://doi.org/10.5209/ rev_CUTS.2014.v27.n1.41727

Scambler, Graham. 2007. «Sex work stigma: opportunist migrants in London». Sociology, 41 (6): 1079-1096. Doi: https://doi.org/10.1177/0038038507082316

Soderlund, Gretchen. 2002. «Covering urban vice: the New York Times, 'white slavery,' and the construction of journalistic knowledge». Critical Studies in Media Communication 19 (4): 438-460. Doi: https://doi.org/10.1080/07393180216567

Solana Ruiz, José Luis y Riopedre, José. 2012. Trabajando en la prostitución: doce relatos de vida. Granada: Comares.

Stenvoll, Dag. 2002. «From Russia with love? Newspaper coverage of cross-border prostitution in Northern Norway, 1990-2000». European Journal of Women's Studies 9 (2): 143-162. Doi: https://doi.org/10.1177/1350682002009002807

Treviño Rangel, Javier. 2009. «Pánico moral en las campañas electorales de 2006. La elaboración del peligro para México». Foro Internacional 49 (3): 638-689. Disponible en el 
enlace http://www.redalyc.org/pdf/599/59921016006.pdf. [Consultado en 9 de marzo de 2017].

Tuchman, Gaye. 1983. La producción de la noticia: Estudios sobre la construcción de la realidad. México: Gustavo Gili.

van Dijk, Teun. 1990. La Noticia como discurso: comprensión, estructura y producción de la información. Barcelona: Paidós.

van Dijk, Teun. 2009. Discurso y poder: contribuciones a los estudios críticos del discurso. Barcelona: Gedisa.

van San, Marion y Bovenkerk, Frank. 2013. «Secret seducers. True tales of pimps in the red light district of Amsterdam». Crime Law and Social Change, 60 (1) : 67-80. Doi: https:// doi.org/10.1007/s10611-013-9436-Z

Ana Belén Puñal Rama. Profesora titular en Comunicación Social en la Universidad Estatal de Milagro (UNEMI) en Ecuador y doctora por la Universidad de Santiago de Compostela. Investigadora en Comunicación y Género, forma parte del grupo de investigación «Comunicación, Ciudadanía y Cambio Social» de la UNEMI y dirige el Observatorio de Comunicación Ciudadana de dicha universidad. Es integrante, así mismo, de la Red Europa-América Latina de Comunicación y Desarrollo.

Ana Tamarit. Profesora titular de la Universidad Estatal de Milagro (Ecuador) y doctora por la Universidad de Salamanca. Investigadora en medios comunitarios, dirige el grupo de investigación «Comunicación, Ciudadanía y Cambio Social» y el Máster en Comunicación, con mención en Medios Comunitarios, de la UNEMI. Codirige, así mismo, el Observatorio de Comunicación Ciudadana de dicha universidad. Forma parte de la Red Europa-América Latina de Comunicación y Desarrollo.

Artículo recibido el 16 de enero de 2017 y aceptado para publicación el 22 de febrero de 2017. 
